\title{
Normatividad en deporte: una reespecificación etnometodológica
}

Normativity in sport: an ethnomethodological reespecification

\author{
Raul SÁNChez García \\ Universidad Europea Madrid \\ raul.sanchez@uem.es (ESPAÑA) \\ Giolo Fele \\ Università degli Studi di Trento \\ giolo.fele@unitn.it (ITALIA)
}

Recibido: 03.09.2013

Aceptado: 23.05.2014

\section{RESUMEN}

Este artículo ofrece una reespecificación del análisis sobre los distintos tipos de normatividad en los juegos deportivos a partir de la etnometodología. En el mundo de la competición deportiva se habla generalmente de dos tipos de reglas: el reglamento y las leyes éticas o fair play. El análisis etnometodológico nos permite además discernir un tercer tipo de normatividad referido a lo que podemos denominar reglas práxicas. El interés de la propuesta etnometodológica no reside en ofrecer un análisis formal (AF) de los distintos tipos de normas deportivas sino más bien en ofrecer análisis detallados del trabajo in vivo de los miembros implicados en casos reales de juego mediante alternos etnometodológicos (AE). Para la elección de casos decidimos realizar una búsqueda en el programa Youtube mediante la introducción de etiquetas tales como "jugada curiosa", "jugada extraña", "trampas", "falta" y "juego sucio" en deporte en general y también en deportes específicos (si bien finalmente acotamos el análisis a los casos de fútbol) en castellano, inglés e italiano. El análisis de las grabaciones permitió una mejor comprensión de la normatividad deportiva, haciendo visible la dimensión práxica y mostrando la compleja articulación entre los distintos tipos de normatividad. 


\title{
PALABRAS CLAVE
}

Etnometodología; Normatividad; Deporte; Reglamento; Fair-play; Reglas práxicas

\begin{abstract}
This paper presents an ethnoethodological reespecification on the analysis of the different kinds of normativity in sport. In sports competition two kinds of rules are normally differentiated: rules of the game and ethical rules or fairplay. The ethnomethodological analysis affords us to distinguish a third kind of normativity, referred to what can be called praxical rules. The interest of the ethnomethodological proposal is not in the formal analysis (FA) of the different types of sport rules but in offering detailed analyses of the in vivo work of the members within real game situations through the use of ethnomethodological alternates (EA). To select the cases of analysis we conducted a search on Youtube , introducing labels such as 'curious play', 'strange play', 'tricks', 'foul', 'dirty play' for sport in general and for specific sports (finally we restricted the cases to football), in Spanish, English and Italian. The analyses of the images allowed us a better comprehension of sport normativity, making visible the praxical dimension and showing the complex articulation among the different kinds of normativities.
\end{abstract}

\section{KEY WORDS}

Ethnomethodology; Normativity; Sport; Rules of the Game; Fair-play; Praxical rules

\section{EL ESTUDIO DEL SENTIDO COMÚN EN LOS JUEGOS DEPORTIVOS}

En el mundo de la competición deportiva se habla generalmente de dos tipos de reglas: el reglamento y las leyes éticas o fair play. El análisis etnometodológico nos va a permitir además discernir un tercer tipo de normatividad referido a lo que podemos denominar reglas práxicas, que determinan lo razonable/no razonable de las acciones respecto a los fundamentos del juego.

Para estudiar la normatividad deportiva en la compleja relación entre sus diversas dimensiones más allá de una clasificación analítica básica debemos estudiar la propia interacción in vivo de los miembros implicados. Para ello vamos a basarnos en las propuestas del programa etnometodológico, cuyo máximo representante es Harold Garfinkel (1963, 1996, 2002, [1967]2006, 2006; Garfinkel y Sacks, 1986). La etnometodología se propone estudiar y 
dar cuenta de los métodos prácticos (etnométodos) que se producen en la acciones concertadas (concerted actions) entre individuos, por los cuales y mediante los cuales se desarrolla un tipo de sentido común (common sense) relacionado con unos patrones normales de acción tal y como se entienden en esa actividad determinada. Esos métodos prácticos son observables-comunicables (accountables), circunstancia referida al hecho de que lo que hacen lo actores cobra sentido (es decir, se puede percibir y se puede hablar sobre ello) para aquéllos con los que se interactúa, ya que, si no, no sería posible ni siquiera esa interacción en la vida social. Esto implica, a su vez, que sean fenómenos que pueden ser estudiados y transmitidos mediante la exposición detallada de los episodios o cursos de acción en los cuales se generan los etnométodos. Este estudio detallado de tales episodios nos permite mantener la temporalidad propia de los cursos de acción, algo que se perdía en otro tipo de análisis tradicionales sobre la actividad física tales como la praxiología de Pierre Parlebas (Parlebas, 2001, 2003; véase Sánchez García, 2011 sobre la relación entre praxiología y etnometodología).

Para Garfinkel las interacciones cotidianas implican unos patrones normales de actividad, un orden concreto generado in situ por los propios miembros presentes en la interacción. Ese fenómeno de orden en construcción atiende siempre a una doble dimensión: (i) fáctica (de los hechos) y (ii) normativa (de los valores). Son los patrones de actividad percibidos como normales los que, al romperse, generan indignación (valoración negativa) en los miembros ocupados en acciones concertadas.

Esta doble dimensión aparece ya en sus escritos tempranos (Garfinkel, 1963) sobre la cuestión de la confianza (trust) como condición de fondo indispensable para que se pueda producir interacción social mutuamente inteligible. Confianza en que tales patrones de acción concertada atienden a una serie de expectativas constitutivas de una normatividad que todo miembro competente de una comunidad específica tiene en cuenta a la hora de interactuar. Garfinkel (1963) utiliza el análisis de juegos como las tres en raya o el ajedrez para ejemplificar el tipo de normatividad que rige las interacciones humanas. Por ejemplo, para el estudio de esa confianza (trust) indispensable en la vida social y de forma más concreta en cualquier juego reglado, realiza el siguiente experimento: jugando al ajedrez, decide en determinados momentos intercambiar la posición en el tablero de varias de sus piezas antes de realizar un movimiento. Según nos cuenta el autor:

"En las ocasiones que hice esto, mi oponente se mostró desconcertado, trató de detenerme, me pidió explicaciones sobre que tramaba, dudaban de la legalidad de la acción (tratando de afirmar su ilegalidad), me hicieron saber que les estaba estropeando el juego y en la siguiente ronda me hicieron prometer que no "haría nada otra vez"” (1963:199).

Es así como, desde la sociología general, es posible encontrar una justificación suficiente para utilizar el análisis de los juegos como terreno acotado privilegiado en el cual estudiar las interacciones que suceden en 
entornos cotidianos de cualquier índole. De hecho, esa es la estrategia utilizada por Garfinkel. Según el autor, las investigaciones sobre las expectativas constitutivas de normatividad "no solo se aplican a las interacciones de juegos sino a las interacciones de la "vida seria" también" (1963:201). Es decir, a partir del estudio de las reglas en los juegos podemos conocer algo más acerca de la actitud natural de los miembros de una colectividad, si bien sin perder nunca de vista que hablamos de un modelo (el de juegos) aplicado de forma restringida a la vida cotidiana, la cual nunca está tan bien delimitada y definida. Teniendo esto en cuenta podemos entender los estudios de autores como Izquierdo (2003) que propone el análisis detallado de los árbitros de fútbol como actividad privilegiada para estudiar ámbitos tan aparentemente dispares como el quehacer científico y más concretamente la metodología en ciencias sociales. Es así también como Kew $(1986,1987,1990,1992)$, siguiendo las indicaciones del programa etnometodológico, investiga la cuestión de las reglas de juego en el deporte $^{1}$ como caso concreto para entender las formas de interacción social.

\section{NORMATIVIDAD EN LOS JUEGOS DESDE UNA APROXIMACIÓN ETNOMETODOLÓGICA}

Según Garfinkel (1963), en los juegos encontramos siempre (A) una serie de reglas básicas (el reglamento) y (B) unas reglas de preferencia de juego. Sobre las (C) reglas éticas, si bien las menciona de pasada, no realiza un desarrollo en profundidad.

A) Las reglas básicas (reglamento) se caracterizan por tres propiedades: (1) desde el punto de vista del jugador, las reglas enmarcan un conjunto de posibilidades que el jugador espera llevar a cabo, independientemente de sus deseos, intereses o consecuencias; (2) el jugador espera que ese mismo conjunto de alternativas posibles se aplique por igual tanto a él como a los demás jugadores; (3) el jugador espera que los demás esperen de él lo mismo que él espera de los demás (p.190). A estas tres propiedades, el autor las denomina expectativas constitutivas. Las reglas de juego constriñen a la vez que permiten la realización de diversas jugadas.

B) Dentro de las posibilidades establecidas por las reglas de reglamento, Garfinkel señala las denominadas como reglas de preferencia de juego (rules of preferred play o rules of preference), que implican una serie de consideraciones sobre "eficacia, eficiencia, preferencia estética, juego convencional, juego precedente, juego tradicional y demás"(Garfinkel, 1963:192). Tales reglas, que

${ }^{1}$ Ejemplos de estudios etnometodológicos sobre la cuestión deportiva, referidos más concretamente a los propios patrones de acción más que a la cuestión de la normatividad se encuentran el clásico estudio realizado en los 60 por McBeth (2006) sobre baloncesto; Fele (1997) sobre las acciones en el fútbol; en Collinson (2006) sobre la actividad de correr juntos como una realización conjunta; Sánchez García (2008) sobre aikido y boxeo; o Coates (1999) en sobre la actividad del boxeo. 
debido a nuestro uso especial de las mismas denominaremos práxicas, ${ }^{2}$ cuya normatividad es del tipo prescriptivo (para obtener tal resultado debes obrar de tal modo) y definen lo que es juego razonable/no razonable desde un criterio de eficiencia. Es a partir de estas reglas como diferenciamos si un jugador es principiante o experto, si es un buen o mal jugador; en definitiva, sobre nivel y calidad de juego.

C) Aparte de los dos tipos de reglas señaladas con anterioridad, debemos tener en cuenta el código ético (fair play). Garfinkel despacha la cuestión diciendo que "las acciones que cumplen las reglas básicas definen en los juegos el "fair play", mientras que los posibles resultados de juego definen la "justicia" (1963:208)". Tal falta de atención a este tipo de esta normatividad en Garfinkel puede ser debido a la cuestión de que su análisis son sobre juegos de mesa y no deportes. En tales juegos de mesa no se hacen tan evidentes los códigos de fair play, algo que en deporte sí se trataba de forma más explícita y son reglas tan o más antiguas que las propias reglas de juego.

La normatividad de los juegos en sus distintas dimensiones se relaciona con el orden constitutivo de eventos de juego (lo que se concibe como juego, como lo que ocurre dentro del juego). Éste está representado por el grupo de posibles eventos al que se asignan las expectativas constitutivas de juego (Garfinkel 1963:191) y es el que permite interpretar y responder a las acciones como acciones de juego y no simples movimientos. ${ }^{3}$ Garfinkel -en gran medida debido a que su análisis se refiere a juegos de mesa (ajedrez o tres en raya) y no a juegos deportivos- se refiere en un primer momento a las expectativas vinculadas a las reglas básicas. Sin embargo, más adelante se refiere al hecho de que el orden constitutivo necesita una regla más que completa la enumeración de reglas básicas, referida a los "términos no expresados del contrato". Esa regla o clausula del acuerdo es la disposición "etcetera" (1963:199). Esta disposición implica unas expectativas constitutivas que también se refieren a las reglas de preferencia (práxicas) y éticas. Según el análisis sobre el artículo original de Garfinkel (1963) que realiza Watson (2009), las expectativas constitutivas de juego se refieren sobre todo a las reglas básicas y en un sentido más débil a las reglas de preferencia de juego. Romper las expectativas de preferencia de juego no amenaza el orden constitutivo de eventos de juego tal y como ocurriría si se rompen respecto a las reglas básicas. Sin embargo, Watson vuelve a dejar fuera de la discusión la normatividad ética, introducida dentro de las reglas de preferencia de juego:

2 Además de las reglas de preferencia de juego, Garfinkel (1963:193) diferencia otro tipo de reglas denominadas condiciones proporcionadas por el juego (game-furnished conditions). Garfinkel apunta la posibilidad de que dentro de las reglas básicas, las estrategias de cualquiera de los jugadores (lo que tanto Livingston (2006:411) como Liberman (2013: 130) denominan como "juego estratégico") pueden basarse en "normas de eficacia instrumental" (Garfinkel, 1963:208) pero no desarrolla más en profundidad esta cuestión. Considero que la acepción de reglas práxicas proporciona mayor claridad para los objetivos perseguidos en la presente investigación.

3 Como señala Garfinkel “...los jugadores de bridge responden a la acción de los demás como eventos de bridge, no como eventos comportamentales" (1963:195). 
“ Tomemos por ejemplo un juego de tenis mixto (mis excusas a los teóricos de juegos por elegir un juego real). Una regla básica es que, un jugador puede golpear con la bola fuerte y directamente al cuerpo del oponente. Sin embargo, una regla de preferencia -en general solo aplicada en tenis jugado en el club- se refiere al hecho de que un jugador no debe golpear directamente a una jugadora. Si lo hace, el árbitro no puede sancionar ese tiro, si bien se desvía de las reglas de preferencia de juego" (2009:280).

Consideramos que en este ejemplo (al igual que ocurre en un partido normal entre jugadores hombres) las expectativas que se están violando tienen que ver con la normatividad ética (sobre cómo relacionarse y tratar al contrario), no con las de preferencia de juego o práxicas, vinculadas al sentido táctico. Desde el sentido táctico, el golpe al cuerpo es perfectamente razonable.

En cualquier caso, de modo general considero que las expectativas constitutivas de juego se aplican a cualquier tipo de normatividad y forman parte de la actitud natural del jugador respecto a lo que constituyen eventos normales de juego normal. Cuando se rompa la normalidad del devenir de los eventos de juego (dimensión fáctica) se hará visible la dimensión normativa en sus distintas acepciones.

\section{METODOLOGÍA}

Para la búsqueda, clasificación y análisis de fenómenos de juego deportivo relacionados con diversas formas de normatividad, hemos decidido adoptar un supuesto metodológico básico de la etnometodología, a saber: la visibilidad del orden social se genera en momentos de ruptura. Esos momentos de ruptura pueden ser generados espontáneamente o pueden ser propiciados por el investigador, como el propio Garfinkel ([1967] 2006) realizaba con sus famosos breaching experiments (experimentos de ruptura), cuya pretensión era "mostrar lo familiar como extraño" (Benson \& Hughes 1991:109). El acceso a tales momentos de ruptura lo hemos realizado a partir del registro de vídeos colgados en Internet mediante buscadores como Youtube. Hasta el momento, la sociología del deporte (y la sociología en general) parece haber pasado por alto la gran utilidad de ese tipo de imágenes sobre cursos de acción natural que ocurren en los juegos deportivos y que son accesibles públicamente. Nos referimos al hecho de que la cantidad y calidad de imágenes y el audio (a veces incluso con transcripciones simultáneas) es muy superior a lo que podamos generar con nuestros propios equipos de registro. No obstante, la cuestión que permanece sin contestar aun es la de establecer criterios para realizar buenas búsquedas. Para el estudio de fenómenos que nos dicen algo sobre la normatividad deportiva decidimos realizar una búsqueda en el programa Youtube mediante la introducción de etiquetas tales como "jugada curiosa", "jugada extraña", "trampas", "falta" y "juego sucio" en deporte en general y también en deportes específicos (fútbol, baloncesto, fútbol americano, atletismo, beisbol) en castellano, inglés e italiano. Estas etiquetas constituyen 
un primer entendimiento, en términos de sentido común, de lo que se considera "anormal", "extraño", "inusual", etc. en comparación con lo que de otro modo pasa inadvertido y no merecedor de ser destacado. De este modo, obtuvimos una primera categorización, realizada por los propios miembros, de los fenómenos destacables por mostrar cierto tipo de desviación respecto al curso normal de los acontecimientos tal y como suceden en los juegos deportivos. Es éste el primer paso necesario en el análisis para una mejor comprensión de la desviación, de la ruptura de la normatividad y los patrones normales de juego.

Tras esta primera búsqueda decidimos acotar el análisis de los casos solo al estudio del fútbol. La razón era simple: ambos investigadores estábamos familiarizados con ese juego y compartíamos con la potencial audiencia española un saber común acerca del juego, debido a que en las tradiciones culturales europeas (al contrario por ejemplo que en EEUU) el fútbol está ampliamente extendido. Este detalle no carece de importancia. El propio Garfinkel animaba a los investigadores a adquirir cierta competencia de los miembros (member competency) para poder explicar de forma más adecuada la actividad que se quería analizar; ésta es la idea fundamental a la hora de llevar a cabo los llamados estudios híbridos (Garfinkel, 1996: 13). No tendría tanto sentido para nosotros tratar de exponer en detalle análisis sobre deportes en los cuales no tenemos tal conocimiento (por ejemplo cricket o fútbol americano) ni tendría tanto sentido exponer tales análisis sobre juegos que en un ámbito cultural como el español no están tan extendidos.

El visionado de las imágenes de fútbol permitió generar las agrupaciones en categorías de diversos fenómenos que guardaban cierta relación de familiaridad en cuanto a su tratamiento desde el punto de vista de las normatividades deportivas. Posteriormente realizamos el análisis detallado del trabajo in vivo de los miembros implicados, tratando de atender a la relevancia de los hechos desde el punto de vista de la normatividad para esos miembros implicados.

\section{ANÁLISIS FORMAL (AF) DE LOS DISTINTOS TIPOS DE NORMATIVIDAD EN DEPORTE}

Para el tratamiento del fenómeno de la normatividad deportiva podemos comenzar desarrollando una clasificación formal que no debe verse más que como un mapa orientativo o cartografía inicial.

\begin{tabular}{|l|l|l|}
\hline $\begin{array}{l}\text { Acciones } \\
\text { Normatividad }\end{array}$ & Acciones aceptadas & Acciones no aceptadas \\
\hline Reglamento & Juego permitido & Falta \\
\hline Ética (Fair play) & Juego respetuoso & Juego sucio \\
\hline Reglas práxicas & Buena jugada & Mala jugada \\
\hline
\end{tabular}

Fig.1 tipos de normatividad y acciones que definen en las actividades deportivas 
Sin embargo, las acciones de juego reales distan mucho de mantenerse tan solo bajo la regulación de un tipo de normatividad. Como veremos en los casos de análisis, la articulación entre el reglamento, el fair play y las reglas práxicas es compleja.

Es decir, las categorías de acciones se relacionan con los distintos tipo de normatividad y engloban una serie de fenómenos que guardan entre sí cierta familiaridad pero ni son cerradas ni agotan de forma exhaustiva y de una vez por todas el corpus original de fenómenos a encontrar. Sería erróneo desde un punto de vista etnometodológico tratar de establecer categorías cerradas, perfectamente definidas en las que enmarcar todos y cada uno de los ejemplos que podemos ir encontrando pensando que así hemos llegado a una definición científica de la normatividad en deporte. Eso sería precisamente confundir la representación analítica del análisis formal (AF) por la realidad objetiva de los hechos sociales (Garfinkel 2002:66). En definitiva, "perder el fenómeno" sobre el cual se investiga. Para remediar tal pérdida presentaremos en el siguiente apartado casos de estudio que representan los alternos etnometodológicos $(\mathrm{AE})^{4}$ sobre normatividad* en deporte: lo legal*, ético* y razonable* y la relación a veces problemática entre ellos se presenta en el trabajo de interacción generado entre los distintos participantes de, en y como orden social; en este caso, el orden* de los juegos deportivos. Precisamente es en esos análisis empíricos detallados del trabajo in vivo de los miembros en los diversos casos que analizaremos podremos encontrar los qués perdidos (missing whats) de las clasificaciones formales (Garfinkel, 2002:99), siempre demasiado rígidas para permitir observar la complejidad y la temporalidad propia de los hechos sociales.

Hecha esta aclaración, presentamos en este momento el análisis formal (AF) de los distintos tipos de normatividad y en el siguiente apartado nos centraremos en los alternos etnometodológicos (AE) para estudiar in situ la relación entre esas normatividades.

Reglamento: también conocido como reglas oficiales o reglas básicas del juego. Sanciona oficialmente las acciones que ocurren durante el juego. En los juegos deportivos, la figura del árbitro vela por el cumplimiento del reglamento. Definen el juego legal o ilegal. Éste es primer nivel de normatividad que normalmente se toma en consideración al referirse al caso de normatividad más obvio: en el juego hay acciones permitidas y otras que están sancionadas. Las 17 normas especificadas en el reglamento del fútbol regulan distintos aspectos del juego, de tal modo que, cogidas en su conjunto, identifican, definen y codifican un juego concreto (fútbol y no por ejemplo rugby). También, algunas de esas reglas prescriben de forma más detallada la realización adecuada de ciertas acciones. Por ejemplo, en la regla 15 sobre el saque de banda leemos sobre el procedimiento:

4 Sobre la relación entre los pares inconmensurables AF (análisis formal) y AE (alternos etnometodológicos) véase Garfinkel 2002:135 y ss). La utilización del * indica que nos encontramos en el análisis propuesto desde los $\mathrm{AE}$, que mantienen la temporalidad de los cursos in vivo de actividad. 
"En el momento de lanzar el balón, el ejecutor deberá:

- $\quad$ estar en frente al terreno de juego

- $\quad$ tener una parte de ambos pies sobre la línea de banda o en el exterior de la misma

- $\quad$ servirse de ambas manos

- lanzar el balón desde detrás y por encima de la cabeza

- lanzar el balón desde el sitio donde salió del terreno de juego

Todos los adversarios deberán permanecer a una distancia que no sea inferior a 2 metros del lugar en que se ejecuta el saque de banda. El balón estará en juego tan pronto haya entrado en el terreno de juego. El ejecutor del saque no deberá volver a jugar el balón hasta que este haya tocado a otro jugador." (FIFA, 2014:48).

Cualquier modo diferente de realizar la acción representaría una infracción y sería sancionado consecuentemente. El encargado principal de hacer velar el cumplimiento de este tipo de normatividad es el árbitro, que es la persona que institucionalmente está a cargo del cumplimiento del reglamento y de penalizar al infractor. En este artículo no estamos interesados en el modo en que los árbitros interpretan las acciones ni en analizar las razones de sus decisiones. Tan solo señalamos el punto de vista institucional conectado con este tipo de normatividad. Es decir, la construcción social intrínseca del mismo a partir de lo institucional: solo es falta cuando es considerado de tal manera por el árbitro. ${ }^{5}$ En este sentido, somos conscientes de que el árbitro puede equivocarse de muy distintas maneras (considerando una falta cuando no ha habido infracción o considerando una acción como adecuada cuando debería haber sido considerada falta, etc.). A esta cuestión nos referimos normalmente con el problema sobre la interpretación de las reglas por parte del árbitro, en particular al problema de la aplicación de una serie de reglas a casos reales que implican acciones sociales (véase sobre esta tema Dal lago, 1990; Coulon, 1998). Nosotros no estamos interesados en esos casos. Nosotros tan solo queremos resaltar el hecho de que las reglas básicas (el reglamento) ofrecen un primer horizonte de expectativas sobre normatividad; que los jugadores se orientan hacia esas expectativas y que el árbitro es la persona que institucionalmente está designada para permitir o sancionar las acciones durante el partido. El árbitro en este caso constituye la fuente (institucional) a partir de la cual la ruptura de las reglas básicas se hace visible y sancionada. Lo demás tan solo cuenta como interpretación (protestas de jugadores que muestran que ha sido sancionado "injustamente", o protestas de jugadores que muestran como han sido tratados de forma injusta respecto a otro adversario que no fue amonestado por la misma acción, etc.) incluso si desde nuestro sentido común del juego cierta injusticia y/o cierto error arbitral ha sido

5 Esta es una versión de la teoría del etiquetado, de forma específica de la visión constitutiva de la desviación (Becker, 1963). Los diferentes puntos de vista sobre lo que ocurrió verdaderamente en el terreno de juego (de los jugadores, de los entrenadores, de los comentaristas deportivos, de los aficionados o del público en general,etc.) constituyen lo que Mel Pollner (1987) denominaba el "modelo ordinario" (mundane model) de la desviación. 
cometido.

Código ético: conocido normalmente como fair play, apela a la deportividad de los comportamientos en el terreno de juego y se refiere a cómo relacionarse y comportarse de manera adecuada con el contrario. Históricamente está vinculado a la noción amateur de los primeros gentlemen que sistematizan y desarrollan el deporte moderno en Inglaterra (fútbol y rugby se desarrollaran en el s.XIX). En esa etapa amateur el deporte se ve más como un modo de adquirir una serie de cualidades y valores caballerosos que como una pura competición donde lo que más importa es el resultado. Por eso, gran parte de esos juegos están gobernados por los códigos del fair play; reglas de etiqueta y comportamiento propio de caballeros. Eso no implica que no hubiera reglamentos escritos. Podemos encontrar ya en 1845 un reglamento sobre el rugby o en 1848 sobre fútbol y es precisamente en la década de 1840 cuando parece producirse la bifurcación de ambas disciplinas que provenían de una rama común (Dunning \& Sheard, 2004). A medida que se profesionalice el juego, el reglamento va a pasar el código sancionador de conductas oficial en el terreno de juego, quedando el fair play como un código informal pero que sigue teniendo influencia en los comportamientos de los jugadores. Básicamente, expresa un código ético que indica que "no todo vale" para ganar; no se puede ganar de cualquier forma y a toda costa. No contiene el poder sancionador del reglamento pero sí es capaz de imponer constricciones al juego. Define el juego ético o no ético. No tiene en el árbitro el representante oficial institucional que lo hace valer como código normativo porque más bien se refiere a una relación entre jugadores, que son los que tienen que hacer valer y poner en práctica ese fair play. El público también aparece como agente colectivo que sanciona o celebra las manifestaciones de juego poco ético o juego ético respectivamente.

Reglas práxicas: son reglas normativas de procedimiento. Determinan cómo hay que proceder para llegar a obtener unos resultados determinados. Las reglas práxicas se refieren a los fundamentos técnicos-tácticos-estratégicos de juego y definen lo que es razonable (lógico o bueno) y lo que no es razonable (ilógico o malo) desde el punto de vista de la eficiencia. Pero, ¿cómo determinamos si una acción representa un juego razonable o por el contrario no razonable? Para ello debemos siempre tener en cuenta la cuestión de los resultados: si los resultados buscados son obtenidos, entonces la acción fue razonable. No obstante, que el resultado sea o no exitoso no depende solo de que la jugada sea o no razonable sino de la ejecución técnica. Si queremos evaluar el sentido táctico sin interferencia de la ejecución técnica debemos tener en cuenta no el resultado final (lo que ocurre) sino el resultado potencial asociado a la intención de la acción: el potencial resultado bueno o malo permitido por la situación. Este criterio de evaluación es precisamente lo que ofrecen las reglas práxicas, las cuales pueden encontrarse en los manuales de entrenamiento específico de cada deporte y sobre los cuales los entrenadores están bien informados. Como principios generales a los que atienden las reglas práxicas en toda disciplina deportiva: 
a) En defensa encontramos simpleza y seguridad: más razonables (mejor) es una acción defensiva cuanto más simple y segura.

b) En ataque encontramos simpleza y amenaza: más razonable (mejor) es una acción ofensiva cuanto más simple y amenazante.

c) En contraataque encontramos simpleza, amenaza y rapidez: más razonable (mejor) es una acción de contraataque cuanto más simple, amenazante y genere rapidez al juego.

Un tratamiento más específico de las reglas práxicas en cada disciplina implicaría analizar las distintas acciones, fases o sistemas de juego. Por ejemplo, si analizamos las reglas práxicas que gobiernan la realización del servicio de tenis podríamos decir que el jugador debe realizar un servicio que trate de buscar el desplazamiento del oponente mediante una dirección y efecto de la bola que le obligue a restar fuera de pista y/o que genere un espacio importante donde dirigir el siguiente golpeo, consiguiendo llevar la iniciativa en el punto (Nielsen \& McPherson, 2001:657).

Muchas veces es fácil ver si el jugador realizó un error en su decisión táctica. La obviedad de tales acciones no es solo transparente a los ojos del entrenador sino a los ojos de un espectador bien informado que conoce el juego. Esto es así porque los que ven el juego lo entienden gracias a esa serie de criterios práxicos que, si bien no siempre son adquiridos de forma explícita, actúan como parte de un trasfondo de interpretación del juego. Es así como podemos apreciar que una acción del jugador fue inteligente o que tenía buena (razonable) intención aunque la jugada no resultó; o que el jugador debía haber pasado pero esperó demasiado y estropeó la jugada al no hacerlo. Por supuesto hay una serie de casos mucho más controvertidos desde el punto de vista práxico: ¿debería haber pasado o regateado? Quizá haya situaciones en las cuales ambas opciones pueden representar un buen sentido del juego. Las acciones potencialmente buenas están ahí en la situación para ser jugadas. Se muestran públicamente en el juego, en la situación social de interacción, en medio del flujo de acciones a medida que el juego se desarrolla.

Resultados: en el apartado anterior sobre reglas práxicas hemos indicado que en la situación real de juego nunca podemos separar la decisión táctica de la ejecución técnica y eso va a tener unas consecuencias específicas sobre los resultados finales de la acción. Según los resultados podemos diferenciar diversos casos:

Buena decisión táctica + buena ejecución técnica $\longrightarrow$ Buen resultado

Un jugador resuelve un 1vs1 contra el portero mediante un regate bien ejecutado.

Buena decisión táctica + mala ejecución técnica $\longrightarrow$ Mal resultado

Un jugador intenta un pase para dejar a un compañero claramente desmarcado pero falla el pase.

Mala decisión táctica + buena ejecución técnica $\longrightarrow$ Buen o mal resultado

a) Buen resultado: imaginamos una situación en la cual el portero ha recibido la pelota del defensa y decide regatear al delantero que ha venido a presionar. 
Si bien en un principio esa acción estaría contra las reglas práxicas que hablan sobre lo razonable en el juego, en ese caso el resultado de la acción es positiva debido a una buena ejecución técnica.

b) Mal resultado: imaginamos que en la misma situación anterior el portero falla en el regate y le marcan gol. En este caso, la decisión táctica y la ejecución técnica fueron malas.

Antes de pasar a analizar casos concretos de análisis en el próximo apartado, deberíamos aclarar nuevamente la relación entre los distintos tipos de normatividad y las expectativas constitutivas de juego. Los eventos constitutivos de juego, lo que se considera como juego normal, se presentan en todo momento relacionados con ciertas expectativas respecto a los distintos tipos de normatividad. El juego normal debe darse dentro unas reglas básicas de reglamento, de acuerdo con un código ético y atendiendo a lo que marcan las reglas de preferencia de juego o práxicas. ${ }^{6}$ En principio, la mayor tensión parecería vivirse entre el código ético (no todo vale para ganar) y el práxico (criterios de eficacia para ganar) dentro de lo que marcan las reglas básicas de juego. Sin embargo, como veremos en los siguientes casos, la articulación entre códigos normativos es más confusa y compleja de lo que cabría esperar a partir de esta primera aproximación analítica a la cuestión.

\section{ESTUDIOS DE CASO (AE) QUE MUESTRAN LA ARTICULACIÓN ENTRE DISTINTOS TIPOS DE NORMATIVIDAD}

El modo en que aparecen en la práctica acciones de juego problemáticas desde el punto de vista de los diversas normatividades es el siguiente: $\left(1^{\circ}\right)$ primero se rompe la normalidad fáctica (la jugada es anormal respecto a los patrones normales de actividad, a las acciones que se encuentran en el juego de modo habitual) lo cual $\left(2^{\circ}\right)$ tiene implicaciones sobre la normatividad (la jugada se entiende como caso de violación de alguna normatividad, que puede ser la del reglamento, la ética del fair-play la de reglas práxicas o combinadas). Depende del caso, los jugadores contrarios apelarán a un código u otro dirigiéndose o bien al representante del reglamento en el campo (el árbitro) o directamente al propio jugador infractor de la normalidad. Puede ocurrir también que los propios compañeros recriminen las acciones de alguien de su mismo equipo que ha violado algún tipo de normatividad (por ejemplo, si esa acción lleva a un desenlace desastroso para el propio equipo). De cualquier modo, a quién se dirigen los jugadores a protestar o a recriminar nos dice mucho sobre el tipo de norma que creen que ha violado. La intensidad de la recriminación viene

6 Como indica A. Rawls "Las partes de una práctica constitutive deben orientarse a las mismas reglas/prácticas [reglamento], debn asumir eso de los otros y asumir que los otros asumen lo mismo de ellos. Los participantes deben además asumir competencia [reglas práxicas] y buenas intenciones [fair play] cada uno de los demás- hasta que los hechos demuestren lo contrario. Si alguno de los requerimientos anteriores fallase, la inteligibilidad mutual también fallaría“" (2009: 368; los corchetes son nuestros). 
además marcada por el resultado (positivo o negativo) de la jugada. Es decir, la protesta es más fuerte si, haciendo esa jugada problemática sacaron beneficio. Si no lo sacaron, hay recriminación pero puede ser también en forma de burla (por ejemplo, porque ha hecho el ridículo).

Veamos ahora cada uno de esos casos problemáticos mediante el análisis detallado de los alternos etnometodológicos (AE) que presentan simultaneidad y/o conflictividad en su regulación mediante distintas normatividades. Por motivos de espacio vamos a mostrar ejemplos que ponen en relación los tipos de normatividad de dos en dos, dejando casos más complejos para futuras investigaciones.

\subsection{Reglas práxicas y reglas éticas (fair play)}

Comencemos con algunos casos obvios. En primer lugar, deberíamos tener en cuenta que la ruptura de las expectativas del rival es la base del juego deportivo. Si tu juego es fácil de esperar tus oponentes estarán en una mejor posición para contrarrestar tus acciones. En un partido entre Brasil y Uruguay, celebrado el 17 de junio de 1970, el famoso jugador Pele corre hacia un balón que un compañero ha pasado adelantado (Fig.2). El portero uruguayo corre hacia el punto en el cual se supone que Pele tomará contacto con el balón para así poder limitar el ángulo de tiro hacia la portería y poder para la posible acción de disparo del delantero.
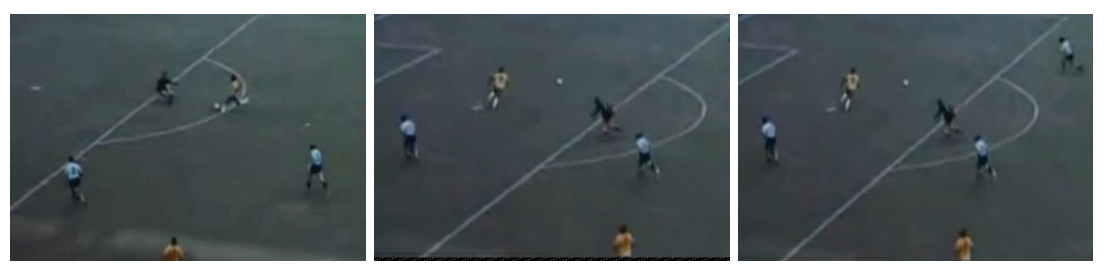

Fig.2 Secuencia de la maniobra del gol de Pele.?

Pele, en vez de tocar el balón, continua corriendo en la misma dirección sin realizar cambio de velocidad o paso. De ese modo sobrepasa al portero por su derecha, dejando que el balón siga su curso y pase al portero por su izquierda. Tras sobrepasar al portero, Pele gira rápidamente hacia la derecha, coge el balón y lo introduce en la portería, marcando gol.

Nadie protestaría por esta forma de engañar al adversario. De hecho, se considera en alta estima que un jugador sea capaz de realizar una jugada tan inesperada para superar al adversario. El caso del caño (túnel, cacha, sotana) es una conocida maniobra para regatear a un oponente haciendo pasar el balón por debajo de las piernas. Ésta acción, si bien tuvo que ser muy sorpresiva en sus primeras realizaciones, es hoy en día utilizada con regularidad como un

7 http://www.youtube.com/watch?v=ryIGWJx CmA 
recurso técnico más y es perfectamente razonable desde el punto de vista de la noramtividad práxica. La acción muestra tanto la habilidad del que la realiza como la responsabilidad del defensor (por no estar suficientemente alerta). Sin embargo, puede ser en ocasiones realizada con la intención de ridiculizar al adversario: tocar el balón para que pase por debajo de las piernas del defensa pueden considerarse en algunos momentos como un acto de falta de respeto. Esta acción por tanto entra dentro de la normatividad práxica pero puede ser controvertida desde el punto de vista ético: lo que para unos es considerado como un uso creativo, inteligente y eficiente de los recursos para solucionar una situación, otros pueden considerarla poco respetuosa. Por tanto, en este tipo de jugadas podemos distinguir dos tipos de expectativas derivadas de dos tipos distintos de normatividad: reglas práxicas y reglas éticas.

Afirmamos que las consideraciones práxicas prevalecerán sobre las éticas en la interpretación de la jugada si la eficiencia de la acción prevalece sobre (o, en caso límite, iguala) su estética. En caso de contradecir esta expectativa, hay más posibilidades de que tome más relevancia interpretativa la normatividad ética y el juego se considere poco ético porque no hay verdadera necesidad de realizarlo. Hablamos de que el jugador se adorna, lo hace de cara a la galería, por lucimiento personal, lo que puede llevar a una actitud irrespetuosa frente al rival.

Tomemos el caso de la Espaldinha de Ronaldo. En el partido entre el Real Madrid y el Atlético de Madrid celebrado el 7 de noviembre de 2010 (Fig.3), Ronaldo lanza a gol desde un lateral y el portero despeja la pelota, que sale bombeada hacia la posición del delantero madridista.

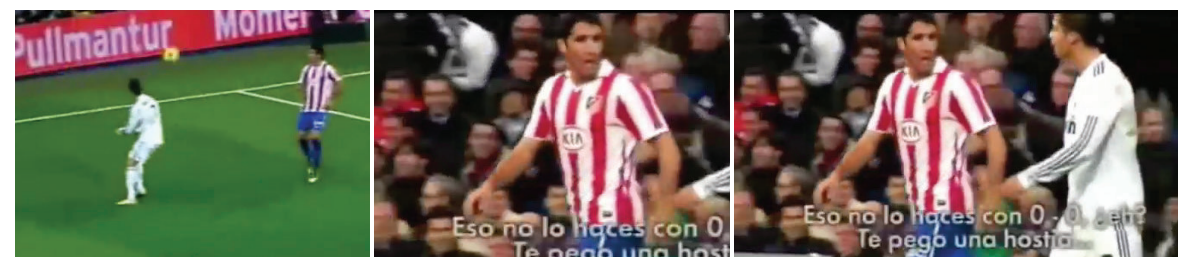

Fig3. Ronaldo realizando la espaldinha en el partido frente al Atlético de Madrid $^{8}$

Éste, en vez de pararla, controlarla o pasarla de un modo convencional (con la cabeza, con la pierna, con el pie), decide en el momento en que la bola va caer darse la vuelta y contactar con la parte superior de la espalda, pasándosela a un compañero. La acción implica un pase del balón sin contacto visual, casi en una actitud de desentendimiento del juego que no representa el cuidado y atención que normalmente se considera en un juego entre futbolistas profesionales. En este caso es dudoso que la eficiencia de la acción prevalezca sobre el adorno del propio jugador. Más aun, esta jugada es considerada poco ética, irrespetuosa para su oponente directo: el defensa Raúl García, que cuando finaliza la jugada se dirige a Ronaldo con las siguientes palabras: "Eso no lo haces con $0-0$, ¿eh?

8 http://www.youtube.com/watch?v=axVF23Khp80 
Te pego una hostia..." Que el defensa haga mención al marcador (el Real Madrid iba ganando 2 goles a 0 a un minuto de acabar el encuentro) deja claro que la gratuitad de la acción se debe a una situación de superioridad manifiesta del Real Madrid en ese punto, lo que añade mayor grado de desconsideración hacia los rivales.

\subsection{Reglamento y reglas éticas (fair play)}

Hay situaciones en las cuales la facticidad normal de las acciones vendría determinada mediante el reglamento pero ocurre algo inusual que tiene implicaciones desde el punto de vista ético, que se erige como normatividad predominante en la regulación del curso de las acciones.

En el partido disputado entre Dinamarca e Irán en 2003, justo antes de finalizar la primera parte, alguien en el público silbó y un jugador iraní, creyendo que el árbitro había pitado final del tiempo reglamentario, coge la pelota con las manos encontrándose dentro del área de meta (Fig 4).A continuación la pasa con el pie hacia el árbitro, como ocurre normalmente cuando acaba el tiempo de juego. La cuestión es que, debido a que el árbitro no había pitado final, el juego era válido y se vio forzado a señalar el penalti a favor de Dinamarca. El árbitro no podía comportarse de otro modo si quería atender a lo que indica el reglamento: era un caso claro de juego deliberado del balón con las manos dentro de la propia área de meta. No había además nada en el reglamento que le permitiera enmendar esa aparente extraña situación. Tras una discusión entre los jugadores, los entrenadores, etc., se toma una decisión. Uno de los jugadores daneses, Morten Weighorst, se dirigió a lanzar el penalti tirándolo de forma deliberada fuera, por la línea de fondo, siendo felicitado por los jugadores iranís y aplaudido por el público.
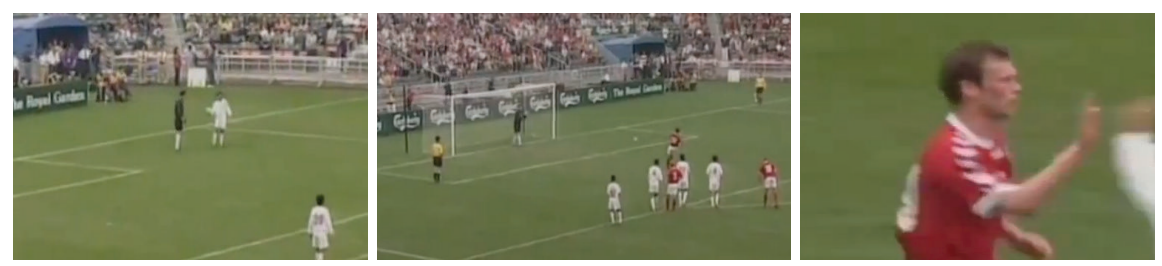

Fig 4. El danés Weighorst fallando a posta el penalti. ${ }^{9}$

El tiro fuera de penalti no se puede considerar una mala ejecución pero sí una acción que está sujeta al reglamento. Al mismo tiempo, no tomar la oportunidad de marcar gol era una forma de respetar la regla no escrita de fair play que indica no tomar ventaja de una situación favorable adquirida en circunstancias fortuitas, cuando los jugadores no tienen responsabilidad en el perjuicio causado a su equipo. La situación sería muy distinta si por ejemplo

9 https://www.youtube.com/watch?v=mKPBIS3 BSo 
un delantero se hallara en una situación ventajosa para marcar tras un error garrafal del portero al fallar un pase de uno de sus compañeros. En este caso, el delantero tiene todo el derecho del mundo (según el reglamento pero también según las reglas éticas) para continuar y marcar, no dando al equipo contrario la posibilidad de enmendar tan craso error.

\subsection{Reglamento y reglas práxicas}

Hay otro tipo de acciones en las cuales se transgrede lo que indica el reglamento y han sido producidas por circunstancias anormales o extrañas, asociadas a cuestiones de tipo práxico (sobre la eficiencia en el juego), que ganan prioridad como normatividad que regula el curso de acción. La falta táctica es el más claro ejemplo de ello: es un acto deliberado de falta, muy diferente a la falta que puede darse en un lance del juego por el control del balón o por una acción mal calculada del defensa frente al atacante (o viceversa). En el caso de la falta táctica, la acción del jugador no se orienta hacia el control del balón sino a dificultar la acción del contrario para que no pueda encontrarse su equipo en posición de clara ventaja. Normalmente son faltas que ocurren por ejemplo en el medio del campo para frenar un posible contraataque. La falta resultante, incluso aunque genere un libre directo o una tarjeta amarilla (o incluso roja) se considera necesaria como mal menor frente a la ventaja que podría obtener el equipo contrario si no se realizara tal acción. Este tipo de acción se denomina táctica precisamente porque se enseña como parte más de los fundamentos defensivos del fútbol y está por tanto sujeta a la normatividad práxica sobre la eficiencia de juego (algo análogo a una maniobra de creación deliberada del fuera de juego, si bien ésta no está penada por el reglamento).

En un encuentro entre Uruguay y Gana para el pase a semifinales de los Mundiales de Suráfrica de 2010, cerca de la finalización de la prórroga (el marcador era 1-1 en esos momentos), hay un remate de cabeza de los jugadores ganeses que es detenido en línea de gol con las manos por el jugador de campo uruguayo Luis Suárez (Fig. 5). El árbitro lo expulsa con tarjeta roja. A continuación el jugador ganés Gyan estrella el balón contra el larguero, fallando el penalti, generando el júbilo en la celebración del propio Luis Suárez. Minutos después se acaba el tiempo reglamentario y Uruguay acabara imponiendose en la tanda de penaltis, consagrando a Suárez como el héroe del partido. 

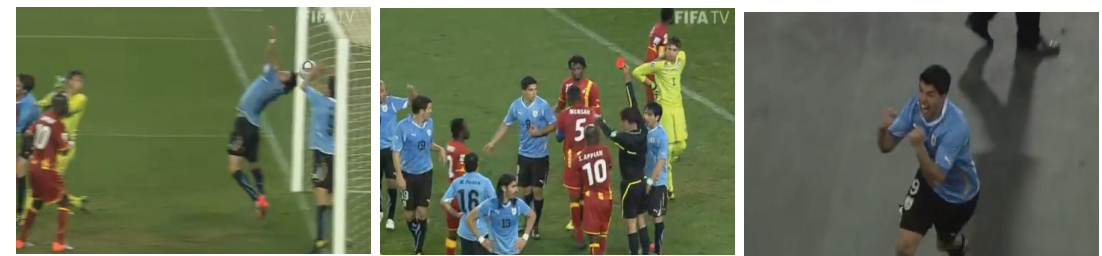

Fig 5. Suárez realizando la falta que generara su expulsión pero que salvará a su equipo. ${ }^{10}$

Este ejemplo, si bien no es una falta táctica muy corriente, sí muestra bien la relación entre el reglamento y la normatividad práxica, imponiéndose esta última debido a la infracción de las reglas de juego como mal menor frente al objetivo final, que era ganar el partido.

\section{CONCLUSIONES Y FUTURAS VÍAS DE INVESTIGACIÓN}

Como principales conclusiones del presente estudio podemos destacar que el enfoque etnometodológico: (1) ha permitido la reespecificación de un sentido más amplio de normatividad a partir de los análisis concretos del trabajo de construcción in vivo de la propia interacción entre miembros que realizan una actividad deportiva. El análisis de las grabaciones hizo visible la, hasta ahora oculta, dimensión práxica y mostró la compleja articulación entre los distintos tipos de normatividad; (2) ha mostrado la doble dimensión del orden de juego: facticidad y normatividad. Una jugada no normal desde el punto de vista de cualquiera de los tres tipos de normatividad se produce en primer lugar (y no a la inversa) por la ruptura fáctica de lo habitual (de los hechos que ocurren normalmente), lo cual tiene consecuencias normativas. En el momento en que se rompen esos patrones normales de acción aparece un caso de violación que remite a uno o varios de los tipos de reglas sancionadoras: reglamento, ética deportiva y reglas práxicas.

Cabe destacar que este artículo tan solo ha sido una primera aproximación a la normatividad deportiva. Como indicábamos en el apartado de análisis, no se han mostrado casos de estudio más complejos como ejemplos que impliquen a la vez los tres tipos de normatividad, algo que se dejará para próximas investigaciones.

Así mismo, no hemos podido prestar atención suficiente atención a la jugada innovadora ni a otro tipo de jugadas que merecen especial atención en sí mismas al romper de modo radical con las expectativas constitutivas de juego: la jugada sin sentido. Estos tipos de jugada pueden además ayudarnos a entender mejor las propias dinámicas de cambio de los juegos deportivos. Por ejemplo, podremos observar cómo la consideración sobre algunas acciones puede cambiar con el tiempo debido a que su relación con algún tipo de normatividad cambia. De jugada innovadora (salto tipo Fosbury) puede pasar a ser simplemente una buena

$10 \quad$ http://www.youtube.com/watch?v=tDpx9GGH79I 
jugada si se adquiere y es usada de forma habitual por los demás jugadores como recurso adecuado y se considera como parte normal del buen juego.

\section{BIBLIOGRAFÍA}

Becker, H. (1963). Outsiders. New York, The Free Press of Glencoe.

Benson, D. \& Hughes, J. A. (1991) "Method: evidence and inference- evidence and inference for ethnomethodology" en Graham Button (ed).

Ethnomethodology and the Human Science, Cambridge, Cambridge University Press, pp. 109-136.

Coates, S. (1999) “Analysing the physical: an ethnomethodological study of boxing”, en Ethnographic Studies, 4, 14-26.

Collinson, J. A. (2006) Running-Together: Some Ethnomethodological Considerations, en Ethnographic Studies, 8.

Coulon, Alain (1998). Le football comme spectacle: le plaisir de l'arbitraire. Communications. 67, 25-32.

Dal Lago, Alessandro (1990). Ermeneutica del calcio. Rassegna italiana di sociologia. 3, 122-141.

Dunning, E. \& Sheard, K. (2004) Barbarians, gentlemen, players. London: Routledge.

Fele, Giolo (1997) "Sociology of Action: ethnomethodological interpretations of soccer", en Analisi de la conversazione e prospettive di ricerca in etnometodologia. Actas del congreso internacional de Urbino, 11-13 julio 1994, 43-58.

FIFA (2014) Reglas de Juego 2013/2014 Zúrich: Fédération Internationale de Football Association.

Garfinkel, Harold (1963) "A Conception of, and Experiments with, "Trust" as aCondition for Stable Concerted Actions", en O.J. Harvey (ed.) Motivation and Social Interaction, New York, Ronald Press, pp. 187-238.

Garfinkel, Harold (1996). "Etnomethodology's Program”, en Social Psychology Quarterly, vol 59, n 1, pp. 5-21.

Garfinkel, Harold (2002) Etnomethodology's Program. Working Out Durkheim's Aforism, Maryland, Rowman and Littlefield.

Garfinkel, Harold [1967](2006). Estudios en etnometodología, Barcelona, Anthropos.

Garfinkel, Harold (2006). Seeing Sociologically, Boulder, Paradigm.

Garfinkel, Harold y Sacks, Harvey (1986). On Formal Structures of Practical Actions, en Harold Garfinkel (ed). Ethnomethodological Studies of Work, London, Routledge, pp. 160-194.

Izquierdo, Antonio J. (2003) “Árbitros de fútbol: ¿un c. elegans para la metodología de las ciencias sociales?”Empiria, 6, pp. 79-111.

Kew, Francis (1986) "Playing the Game: an ethnomethodological perspective", enInternational Review for the Sociology of Sport, 21,4,305-322.

Kew, Francis, (1987): "Contested rules: an explanation of how games change", International Review for the Sociology of Sport, 22, 2,122-135.

Kew, Francis (1990): "The development of games: an endogenous explanation".

International Review for the Sociology of Sport, 25,4, 251-267.

Kew, Francis (1992) "Game-rules and social theory."International Review for the Sociology of Sport 27,4, 293-307.

Liberman, K. (2013). More Studies in Ethnomethodology. New York: SUNY Press.

Livingston, E. (2006). "Ethnomethodological studies of mediated interaction and 
mundane expertise". The Sociological Review, 54(3), 405-425.

MacBeth, D. (2006) "Some notes on the play of basketball in it scircumstancial detail, and an introduction to their occasion" en Human Studies, 35,2, 193-208.

Nielsen, T. M. y McPherson, S. L. (2001). Response selection and execution skills of professionals and novices during singles tennis competition. Perceptual and Motor Skills, 93, 541-555.

Parlebas, P. ( 2001 ) Léxico de praxiología motriz. Barcelona: Paidotribo.

Parlebas, P. ( 2003 ) Elementos de Sociología del Deporte. Málaga: IDA.

Pollner, M. (1987). Mundane Reason. Cambridge, CUP.

Rawls, A.W. (2009). Editor's Introduction. Journal of Classical Sociology, 9, 357-370.

Sánchez García, R. (2008) "Análisis etnometodológico del dinamismo del concepto de habitus en Bourdieu y en Elias en el desarrollo de actividades corporales", REIS, 124, pp. 209-231.

Sánchez García, R. (2011). Para una crítica epistemológica de la praxiología motriz. Apunts, 104,pp.11-20.

Watson, R. (2009) “Constitutive practices and Garfinkel's notion of trust: revisited”, en Journal of Classical Sociology, 9,4, pp. 475-499. 
